\title{
Mixing Loops and Scattered Correlations Produced by Mixing of Melts Derived from a Spatially Heterogenous Mantle
}

\author{
YAN LIANG
}

Brown University

Presenting Author: yan_liang@brown.edu

Variations of radiogenic isotope ratios and highly incompatible trace elements in basalts have generally been attributed to mixing. In an isotope ratio correlation diagram, magmas produced by mixing of melts derived from an enriched mantle (EM) and a depleted mantle (DM) define a hyperbola. Given the proportion of enriched and depleted melts, isotope composition of well-mixed melt is uniquely determined and plotted as a point on the mixing hyperbola. The binary mixing model is constructed through mass balance and does not consider the physical state and spatial distribution of the two endmember components in the mantle source. The geometry of the enriched mantle, such as its size and spatial distribution, exerts a strong influence on the variations of radiogenic isotope and highly incompatible trace element ratios in melts produced by melting a spatially heterogeneous mantle. The purpose of this study is to integrate spatial distribution of mantle heterogeneity into geochemical mixing models. We present a four-stage melting model that takes into account the spatial location and evolution stage of an EM parcel in the melting column. We show how $\mathrm{Sr}$, $\mathrm{Nd}$, and $\mathrm{Hf}$ concentrations and isotope ratios in the pooled melt vary as a function of EM location in the melting column. With changing EM location in the melting column, compositions of the well-mixed pooled melt do not follow a single mixing curve expected by the binary mixing model. Instead, they define a mixing loop that has an enriched branch and a depleted branch in composition space. Figure 1 shows an example of $\mathrm{Sr}-\mathrm{Nd}$ in the pooled melt. The origin of the mixing loop can be traced back to four types EM location or configurations in the melting column. The shape of the mixing loop depends on the relative melting rate of EM to that of DM and the number and spacing of EM parcels in the melting column. As an application, we consider correlations in ${ }^{87} \mathrm{Sr} /{ }^{86} \mathrm{Sr}$ vs. ${ }^{143} \mathrm{Nd} /{ }^{144} \mathrm{Nd}$ and ${ }^{143} \mathrm{Nd} /{ }^{144} \mathrm{Nd}$ vs. ${ }^{176} \mathrm{Hf} /{ }^{177} \mathrm{Hf}$ in oceanic basalts. We show that the scattered correlations in the isotope ratio diagram arise naturally from melting of a spatially heterogeneous mantle.
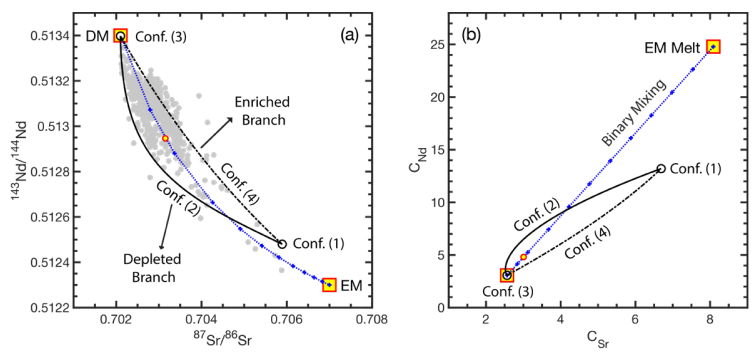